\title{
IMPLANTAÇÃO DO PROTOCOLO PREVENTIVO DE BRONCOASPIRAÇÃO EM UM HOSPITAL PÚBLICO DO ESTADO DO PIAUÍ DE ALTA COMPLEXIDADE: RELATO DE EXPERIÊNCIA
}

\author{
IMPLEMENTATION OF THE BRONCHOASPIRATION PREVENTIVE \\ PROTOCOL IN A PUBLIC HOSPITAL IN THE STATE OF PIAUÍ OF \\ HIGH COMPLEXITY: EXPERIENCE REPORT
}

Manuela Moura de Souza Félix ${ }^{1}$

Wladilleny Cerqueira Lemos Viana ${ }^{2}$

RESUMO: Objetivo: Descrever o processo de implantação do Protocolo Preventivo de Broncoaspiração Multiprofissional em um Hospital Público do Estado do Piauí de Alta complexidade. Método: Este estudo constitui um relato de experiência, de caráter retrospectivo e descritivo, da implantação do Protocolo Preventivo de Broncoaspiração com ações multiprofissionais, pelo Serviço de Fonoaudiologia do hospital. Os dados referentes à sinalização do risco de Broncoaspiração e atendimento dos pacientes foram coletados através das avaliações fonoaudiológicas realizadas na Unidade de Terapia Intensiva geral do referido hospital. Resultados: Sinalizaram-se 134 pacientes com a placa "nada por via oral", nas UTIs, no ano de 2020, consistindo em uma das medidas preventivas essenciais que inibe a oferta por via oral de alimentos, água e medicamentos a pacientes com risco de broncoaspiração. Além disso, iniciou-se o acompanhamento e reabilitação dos pacientes. É oportuno ressaltar que, apesar das dificuldades com a pandemia, obteve-se êxito com uma aplicação adaptada e parcial do protocolo, com medidas simples de segurança tanto para profissionais quanto para os pacientes. Conclusão: A experiência relatada aponta que um trabalho preventivo realizado por uma equipe multiprofissional capacitada e engajada impacta diretamente na qualidade da assistência prestada à população, com boas práticas de cuidado e melhor manejo das alterações da deglutição, proporcionando qualidade de vida aos pacientes, redução do tempo de internação e diminuição dos custos hospitalares,

\footnotetext{
1 Fonoaudióloga. Especialista em Motricidade Orofacial com Enfoque em Disfagia no Âmbito Hospitalar. Fonoaudióloga. Hospital Getúlio Vargas. Teresina, Piauí, Brasil. E-mail: fgamanuelamoura27@gmail.com Autor para correspondência - Endereço: Av. Presidente Kennedy, 8200. CEP: 64063010.

${ }^{2}$ Fonoaudióloga. Especialista em Fonoaudiologia Hospitalar. Fonoaudióloga. Hospital Getúlio Vargas. Teresina,Piauí, Brasil. E-mail: wladylemos@hotmail.com.
} 
mesmo diante de dificuldades no processo de implantação e gerenciamento do Protocolo Preventivo de Broncoaspiração, gerados principalmente pela pandemia da Covid-19.

Palavras chave: Transtornos de Deglutição. Fonoaudiologia. Equipe de Assistência ao Paciente. Protocolos. Segurança.

ABSTRACT: Objective: To describe the implementation process of the Preventive Protocol for Multiprofessional Bronchoaspiration in a Public Hospital in the State of Piaui of high complexity. Method: This study is an experience report, retrospective and descriptive, of the implementation of the Bronchial Aspiration Preventive Protocol with multiprofessional actions, by the Speech Therapy Service of the hospital. The data referring to the signaling of the risk of Bronchoaspiration and patient care were collected through speech therapy evaluations carried out in the general Intensive Care Unit of the hospital. Results: 134 patients were signaled with the plaque "nothing by mouth", in the ICUs, in 2020, consisting of one of the essential preventive measures that inhibits the oral offer of food, water and medication to patients at risk of bronchoaspiration. In addition, monitoring and rehabilitation of patients began. It is worth mentioning that, despite the difficulties with the pandemic, it was successful with an adapted and partial application of the protocol, with simple safety measures for both professionals and patients. Conclusion: The reported experience points out that preventive work carried out by a trained and engaged multiprofessional team directly impacts the quality of care provided to the population, with good care practices and better handling of swallowing changes, providing quality of life to patients, reducing hospitalization time and decrease in hospital costs, even in the face of difficulties in the process of implantation and management of the Bronchial Aspiration Preventive Protocol, generated mainly by the Covid-19 pandemic.

Keywords: Deglutition Disorders. Speech, Language and Hearing Sciences. Patient Care Team. Protocols. Safety. 


\section{INTRODUÇÃO}

A Organização Mundial de Saúde (OMS) reconhece a Disfagia Orofaríngea na Classificação Internacional de Doenças (C.I.D.). Ela afeta aproximadamente mais de $30 \%$ dos pacientes com acidente vascular cerebral (AVC), $52 \%$ a $82 \%$ dos pacientes com doença de Parkinson, $84 \%$ dos pacientes com Alzheimer, $40 \%$ dos adultos acima de 65 anos de idade e mais de $60 \%$ dos idosos institucionalizados (LAGO, 2020).

Como afirma Marchesan (1995) a disfagia pode resultar de uma anormalidade anatômica ou funcional em qualquer estrutura e fase do processo de deglutição. A gravidade da disfagia orofaríngea varia da leve até a completa incapacidade de engolir. A diminuição na eficácia da deglutição pode levar o paciente à desnutrição e à desidratação. Além disso, a diminuição na segurança da deglutição pode causar asfixia e aspiração traqueobrônquica, o que resulta em pneumonia em $50 \%$ dos casos, com uma taxa de mortalidade associada de até 50\% (CLAVE; VERDAGUER; ARREOLA, 2005).

Arnold (2016), Ko (2015) consideram que no ambiente hospitalar, a prevalência de disfagia pode variar entre $20 \%$ e $60 \%$ dos casos, dependendo da distribuição das causas de internação, das comorbidades associadas e das características da população atendida. Em pacientes hospitalizados, a rápida identificação dos disfágicos está relacionada à redução do risco de pneumonia, menor tempo de permanência hospitalar e melhor relação custo-eficácia, resultante da diminuição dos dias de internação (MARTINO; PRON; DIAMANT, 2000).

A dificuldade de deglutir, assim como os distúrbios gastrointestinais, são pilares da broncoaspiração. Conforme ANVISA (2020), e Conselho Federal de Fonoaudiologia (2020) Esse evento ocorre pela infiltração de partículas alimentares, de fluidos da orofaringe ou de conteúdos gástricos em vias aéreas inferiores, podendo desencadear pneumonia infecciosa, pneumonite química, síndrome da angústia respiratória, com dispnéia e dessaturações agudas, desidratação e 
desnutrição. Salienta-se que, em alguns casos, há necessidade de reintubação e de uso de via alternativa de alimentação, com impacto na qualidade de vida e, nos casos mais graves, com aumento do risco de óbito, ANVISA (2020), Conselho Federal de Fonoaudiologia (2020) e Favero (2017).

No ambiente hospitalar, seja nas enfermarias ou na UTI, existem várias situações que aumentam o risco de broncoaspiração por disfagia: de refluxo/êmese (conteúdo baixo), assim como rebaixamento do nível de consciência. As causas mais comuns são por instabilidade clínica: excesso de analgesia, sedação ou uso de bloqueadores neuromusculares; distúrbios hidroeletrolíticos, quadros de sepse; imobilidade e fraqueza adquirida; sarcopenia, presença de via aérea artificial por mais de 48h, presença de sonda nasogástrica, além do prejuízo com higiene oral.

Nesse sentido, existem várias medidas de prevenção que podem ser tomadas visando amenizar esse risco. Os profissionais da equipe multidisciplinar - médicos, enfermeiros e técnicos de enfermagem, fisioterapeutas, nutricionistas, fonoaudiólogos, psicólogos, farmacêuticos e assistentes sociais juntamente com Núcleo de Segurança e $\mathrm{CCIH} \mathrm{-} \mathrm{podem} \mathrm{realizar} \mathrm{ações} \mathrm{preventivas} \mathrm{visando} \mathrm{à}$ diminuição das ocorrências de broncoaspiração e à minimização dos riscos aos quais o paciente está exposto (ALMEIDA, 2016). Por exemplo, estudos sugerem que a higiene bucal diária para pacientes alimentados por sondas reduziu consideravelmente a incidência de pneumonia (FUENTE, 2016). Ademais, modificações na dieta com adequações de acordo com a criticidade do paciente também estão entre as medidas preventivas, assim como a elevação da cama em $45^{\circ}$ é item básico dos cuidados com pacientes de risco (BAINE; YU; SUMME, 2001).

Como citam Fuller (2009), Marik (2001) a ocorrência de broncoaspiração já foi bastante documentada em diversos países do mundo e é um dos principais eventos adversos que atingem pacientes internados. No Brasil, cujos dados são recentes, em um período de um ano foram notificados ao Sistema Nacional de Vigilância Sanitária (SNVS) 86 óbitos de pacientes causados por aspiração broncopulmonar, representando $9 \%$ do total de óbitos decorrentes de eventos adversos neste período, tornando-se a segunda causa de morte, atrás apenas das falhas durante a assistência a saúde (ANVISA, 2020). 
Deste modo, a Fonoaudiologia e a equipe multiprofissional possuem um relevante papel na detecção precoce da disfagia, de distúrbios gastrointestinais e demais afecções que aumentam o risco de broncoaspiração. As intervenções preventivas são fundamentais noâmbito hospitalar, a fim de melhorar a qualidade do cuidado e a segurança do paciente, além de favorecer a diminuição de custos de saúde (NASCIMENTO, 2019). Nesse contexto, este estudo tem por objetivo descrever a criação e implantação do Protocolo Preventivo de Broncoaspiração multiprofissional em um hospital público estadual de alta complexidade.

\section{MÉTODO}

Trata-se de estudo do tipo relato de experiência, de caráter retrospectivo e descritivo, referente à criação e implantação do Protocolo Preventivo de Broncoaspiração Multiprofissional em um Hospital Público do Estado do Piauí de alta complexidade, no ano de 2020. A instituição hospitalar é referência em várias especialidades médicas e recebe pacientes pela central de regulação do SUS, provenientes de hospitais municipais, do interior do Estado e de outros estados circunvizinhos, sendo considerado um dos maiores hospitais da região Meio Norte do país.

O hospital é composto por duas Unidades de Terapia Intensiva - UTI Geral com dez leitos cada, trinta leitos de UTI exclusivos para pacientes com Covid-19 (chegou-se aonúmero de cinquenta leitos durante a pandemia), trezentos leitos de enfermaria, dos quais trinta são exclusivos para internação de pacientes com Covid19. As principais especialidades médicas são neurologia, clínica médica, cardiologia, pneumologia, clínica cirúrgica,ortopedia, nefrologia e dermatologia.

A atuação fonoaudiológica no hospital é recente e o serviço encontra-se em processo de implantação. Conta-se com duas fonoaudiólogas, sendo uma exclusiva para as UTIs e outra para as enfermarias (esta última atende especialmente às solicitações de parecer e acompanhamento dos pacientes que saem da UTI). Com o aumento do número de pacientes na pandemia, as duas se revezaram nos 
atendimentos aos pacientes com Covid-19. Diante disso, considerando que o hospital é uma instituição que preza pela segurança do paciente e qualidade da assistência, criou-se um Protocolo Preventivo de Broncoaspiração de seguimento multiprofissional, em que os fatores de risco para disfagia orofaríngea são identificados na admissão do paciente pela equipe de enfermagem, com solicitação de avaliação especializada pelo fonoaudiólogo em até vinte e quatro horas após a admissão ou durante a internação.

A implantação do protocolo, além de garantir um seguimento multiprofissional com melhor suporte às sequelas da deglutição, aliado às boas práticas do cuidado, fortalece o serviço de fonoaudiologia e a atuação da especialidade no contexto hospitalar. É oportuno ressaltar que, com dados do Protocolo, poder-se-ia sensibilizar os gestores da necessidade de contratação de mais profissionais. Salienta-se que, na literatura, não há registro da presença desse protocolo nas instituições públicas hospitalares em todo o Estado. Destaca-se também que a ANVISA lançou a nota técnica 10/2020 a qual trata de práticas seguras para a prevenção de aspiração broncopulmonar em serviços de saúde, reforçando a importância da implantação deste protocolo com ações multiprofissionais, notificação de óbitos em decorrência de broncoaspiração e monitoramento de indicadores.

\section{RESULTADOS}

O Protocolo Preventivo de Broncoaspiração foi planejado desde o início dos atendimentos fonoaudiológicos no hospital, no ano de 2014. Porém, a grande maioria dos profissionais desconhecia a atuação fonoaudiológica, e as ações específicas eram realizadas por outros profissionais da assistência, sem a devida especialização.

Observou-se que os pacientes permaneciam por muito tempo com uso de sonda nasoenteral para alimentação, em razão da ausência do fonoaudiólogo, o qual propiciaria a reabilitação da via oral segura (Conselho Federal de Fonoaudiologia, 2020). Apurou-se também que os demais profissionais realizavam 
tentativas improvisadas de alimentação dos pacientes com fatores de risco para disfagia, ofertando alimentos em consistências inapropriadas, além de água e outros líquidos ("dieta líquida de prova"). Por exemplo, verificou-se a presença de café, bolo e outros gêneros alimentícios no traqueóstomo dos pacientes.

Nesse contexto, salienta-se que, conforme Pires et al (2012), o líquido é a consistência que mais traz risco a esses pacientes, já que eles apresentam tanto a alteração de sensibilidade intraoral e de motricidade orofacial, como a diminuição da proteção de vias aéreas. Desta forma, impede-se o controle intraoral adequado, o que aumenta a probabilidade de penetração, aspiração e broncoaspiração.

Em vista disso, o trabalho inicial do serviço de Fonoaudiologia do hospital foi possibilitar a compreensão dos demais profissionais acerca da atuação e da importância do trabalho fonoaudiológico com os pacientes na UTI que apresentavam risco para disfagia. Além disso, efetuou-se a busca ativa para avaliação e indicação da consistência alimentar mais adequada a cada caso ou de via alternativa de alimentação, a curto e longo prazo, junto à equipe multiprofissional.

Quando encerrados os atendimentos na UTI, realizava-se busca ativa na clínica neurológica do hospital, a qual apresentava grande demanda de pacientes em situação grave, com sequelas, em longo período de internação e com uso prolongado de sonda nasoenteral. Comumente se admitiam, na clínica neurológica, pacientes do interior do Estado com anos de uso dessa sonda de alimentação.

A resposta foi bastante positiva tanto na UTI quanto na clínica neurológica, vez que os pacientes começavam a evoluir dentro de suas possibilidades reais. Quando não era possível uma alimentação por via oral, sugeria-se a realização da gastrostomia, a qual consiste no procedimento mais indicado para pacientes que necessitam de um processo de reabilitação maior ou com impossibilidade de retorno da via oral (JOTZ, G. P et al, 2009).

Com respostas positivas e rápidas, os médicos das demais clínicas passaram a solicitar cada vez mais pareceres, tornando o serviço mais demandado. Alguns anos depois foi contratada uma segunda fonoaudióloga, quando então se concretizou a implantação do protocolo com o apoio da equipe multiprofissional. Em outubro de 2019, apresentou-se o projeto à alta gestão, o qual foi prontamente aprovado. Assim, desencadearam-se a implantação (com os devidos ajustes), a 
confecção de materiais, folders, cartazes, placas, fluxogramas (figura 1), além do treinamento das equipes, com monitoramento de taxas e indicadores.

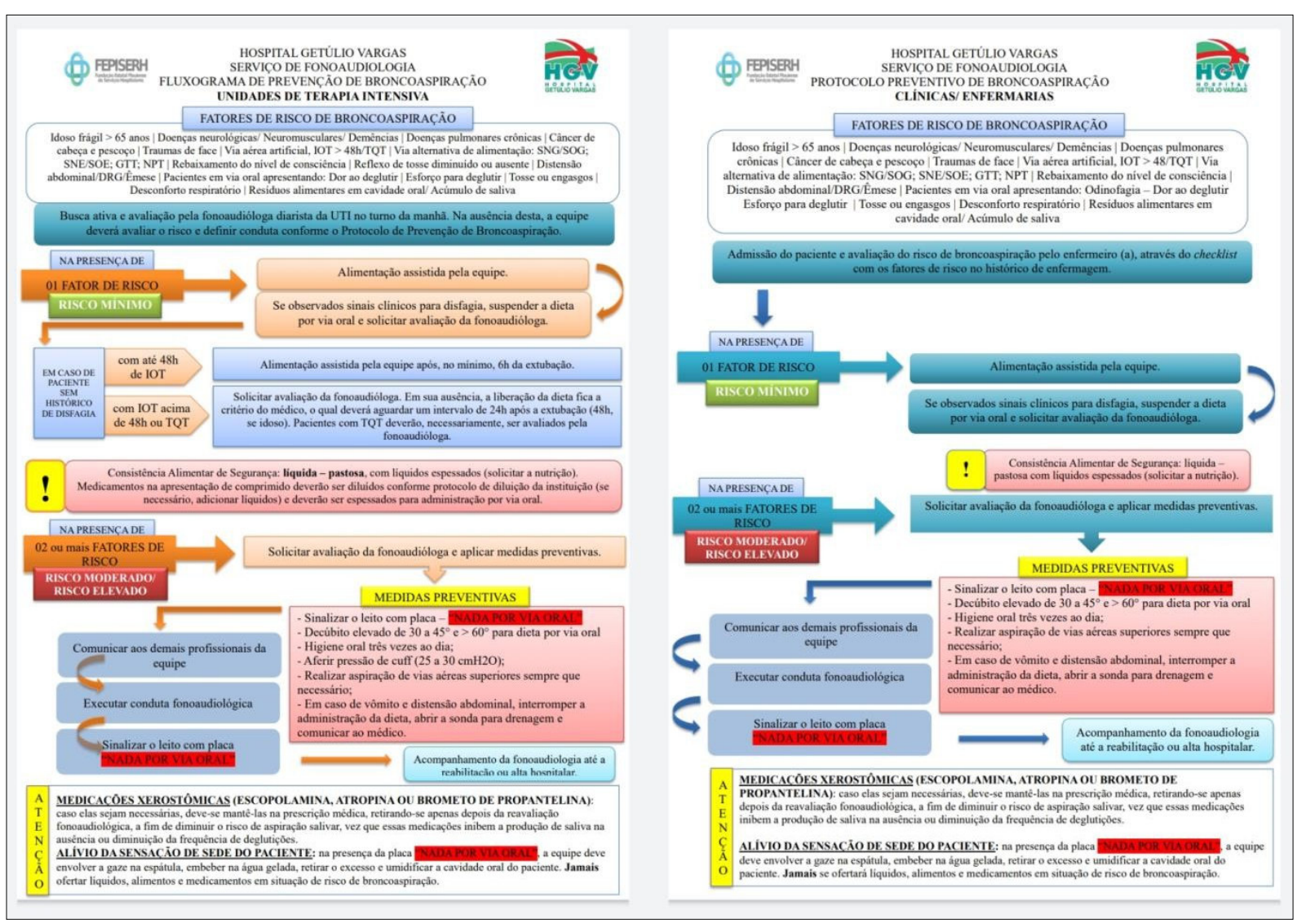

Figura 1. Fluxogramas relativos ao Protocolo Preventivo de Broncoaspiração.

Enfatiza-se que o protocolo foi criado e adaptado à realidade do serviço de fonoaudiologia daquela instituição, tratando-se de uma circunstância determinante para a escolha da associação de dois fatores de risco para a solicitação da avaliação fonoaudiológica especializada. A presença da fonoaudióloga na constatação de apenas um fator de risco, conforme preconiza a literatura, seria inviável em razão da alta demanda do hospital.

Em 2020, deu-se início aos treinamentos pelas UTIs. Contudo, o protocolo prevê metas de cobertura de 80 a $100 \%$ da equipe treinada a identificar os riscos, eliminar os danose estabelecer medidas preventivas adequadas, atendendo a todos que são classificados com risco para disfagia e broncoaspiração. Nos setores das enfermarias, os treinamentos também foram iniciados, porém houve demora na 
adequação do histórico de enfermagem, documento em que o checklist com os riscos de broncoaspiração seriam anexados e o enfermeiro avaliaria o risco na admissão e solicitaria avaliação fonoaudiológica especializada em 24h. Com o advento da pandemia de Covid-19, houve uma pausa nos treinamentos, os quais retornaram em 2021. Nessa fase, esses foram continuados com o checklist já anexado ao prontuário eletrônico.

Destaca-se que, nesse processo de educação continuada, foi imprescindível o apoioda alta gestão do hospital, bem como dos núcleos de educação permanente, de segurança do paciente, da comissão de controle de infecção hospitalar e da equipe multiprofissional, formada por médicos, enfermeiros, técnicos de enfermagem, fisioterapeutas, nutricionistas, fonoaudiólogos, psicólogos, farmacêuticos, odontólogos e assistentes sociais.

Naquele ano de 2020, foram sinalizados 134 pacientes com a placa "nada por via oral" (figura 2), uma das medidas preventivas essenciais que inibe oferta por via oral de alimentos, água e medicamentos a pacientes com risco de broncoaspiração. Além disso, iniciou-se o processo de reabilitação destes pacientes. É oportuno ressaltar que, devido à pandemia, houve dificuldades com treinamentos e gerenciamento do protocolo. Apesar disso, verificou-se êxito com uma aplicação adaptada e parcial do protocolo, com medidas simples de segurança tanto para profissionais quanto para os pacientes, sendo multiprofissional e de baixo custo, proporcionando melhoria da qualidade de vida aos pacientes e diminuição de custos hospitalares. 


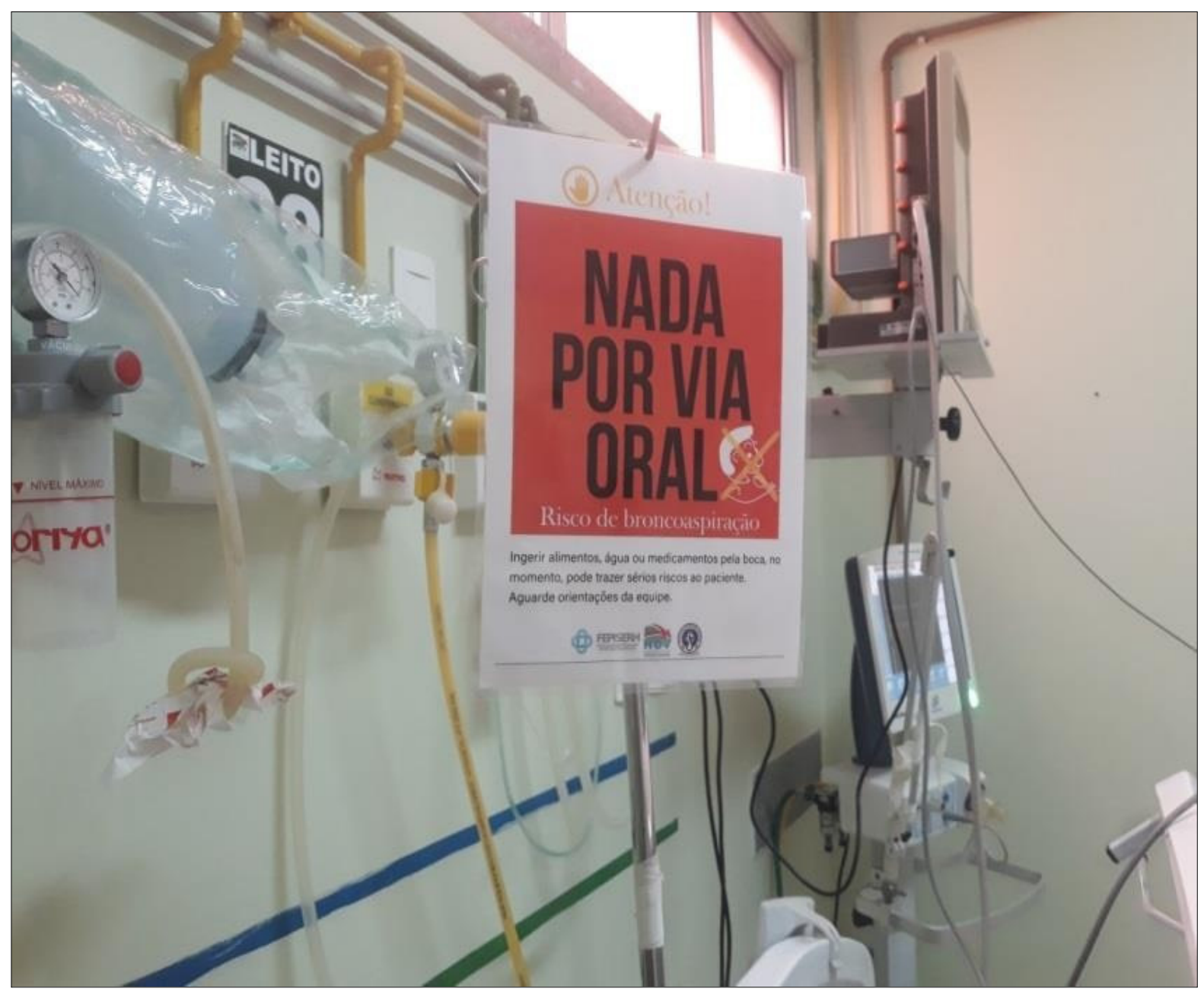

Figura 2. Disposição no leito da placa sinalizadora "nada por via oral", em razão do risco debroncoaspiração.

\section{CONCLUSÃO}

A experiência relatada aponta que um trabalho preventivo realizado por uma equipe multiprofissional capacitada e engajada impacta diretamente na qualidade da assistência prestada à população, com boas práticas de cuidado e melhor manejo das alterações da deglutição, proporcionando qualidade de vida aos pacientes, redução do tempo de internação e diminuição dos custos hospitalares, mesmo 
diante de dificuldades no processo de implantação e gerenciamento do Protocolo Preventivo de Broncoaspiração, gerados principalmente pela pandemia da Covid-19.

\section{REFERÊNCIAS BIBLIOGRÁFICAS}

ALMEIDA, A. E. M. et al. Prevalência de risco moderado e alto de aspiração em pacientes hospitalizados e custo-efetividade da aplicação de protocolo preventivo. J Bras Econ Saúde,v. 8, n. $3, \quad$ p. 216-220, 2016. Disponível em: <https://docs.bvsalud.org/biblioref/2017/02/831902/jbes83-ao6-prevalencia-de-risco- moderadoe-alto-de-aspiracao.pdf>. Acesso em: 15. fev. 2020.

ARNOLD, M. et al. Dysphagia in acute stroke: Incidence, burden and impact on clinical outcome. PLoS One. 2016. 11p. Disponível em: <http://dx.doi.org/10.1371/journal.pone.0148424>. Acesso em: 16. fev. 2020.

BAINE, W. B.; Y. U. W.; SUMME, J. P. Epidemiologic trends in the hospitalization of elderly Medicare patients for pneumonia, 1991-1998. Am J Public Health, v. 91, n. 7, p. 1121-1123,jul. 2001.

BRASIL. AGENNCIA NACIONAL DE VIGILÂNCIA SANITÁRIA. Práticas seguras para prevenção de aspiração broncopulmonar em serviços de saúde. Brasília: ANVISA, 2020.

BRASIL. CONSELHO FEDERAL DE FONOAUDIOLOGIA. "Dispõe sobre a atuação do fonoaudiólogo na equipe multiprofissional na prevenção de broncoaspiração". Parecer CFFa no 48, de 02 de outubro de 2020.

CLAVE, P.; VERDAGUER, A.; ARREOLA, V. Oral - pharyngeal dysphagia in the elderly. Medicina Clinica, v. 124, n. 19, p. 742-748, may. 2005.

FAVERO, S. R. et al. Complicações clínicas da disfagia em pacientes internados em umaUTI. Distúrb Comun, São Paulo, v. 29, n. 4, p. 654-662, dez. 2017.

FUENTE. et al. Enteral nutrition in patients receiving mechanical ventilation in a proneposition. JPEN J Parenter Enteral Nutr, v. 40, n. 2, p. 250-255, 2016.

FULLER. et al. Estimating the costs of potentially preventable hospital acquiredcomplications. Health Care Financ Rev, v. 30, n. 4, p. 17-32, 2009.

JOTZ, G. P. et al. Traqueostomias e Sondas Nasogástricas e Enterais - Implicações na deglutição. In: JOTZ, G. P.; ANGELIS, E. C.; BARROS, A. P. B. Tratado da Deglutição e Disfagia no adulto e na criança. São Paulo: Revinter, 2009.

$\mathrm{KO}$, L. et al. Prevalence of dysphagia in patients with head and neck cancer at dental clinic. Hospital USM. J Sch Dent Sci, v. 10, n. 1, p. 10-16, 2015.

LAGO, F. Terapia Nutricional em Disfagia: Ingestão Segura de Alimentos para maisQualidade de Vida. mar. 2020. Disponível em: <https://www.avantenestle.com.br/sites/default/files/202003/lamina_artigo_disfagia_HCP_0.pdf>. Acesso em: 14. Fev. 2021.

MARCHESAN, I. A. Disfagia. In: Tópicos de fonoaudiologia. São Paulo: Lovise; 1995. MARIK, P. E. Aspiration pneumonitis and aspiration pneumonia. N Engl J Med, v. 344, n. 9, p. 665-671, 2001. 
MARTINO, R.; PRON, G.; DIAMANT, N. Screening for Oropharyngeal Dysphagia in Stroke: Insufficient Evidence for Guidelines. Dysphagia, v. 15, n. 1, p. 19-30, 2000.

NASCIMENTO, J. R. A. Importância do Fonoaudiólogo no protocolo Prevenção de Pneumonia aspirativa na Unidade de Terapia Intensiva. Arquivos Disfagia Fresenius Kabi, 2019.

<https://www.amib.org.br/fileadmin/user_upload/amib/disfagia2019/Informe-Disfagia.pdf>.

Acesso em: 10. jan. 2020.

PIRES, Elaine Cristina et al. Alimentos na consistência líquida e deglutição: uma revisãocrítica da literatura. Rev. soc. bras. fonoaudiol. São Paulo, v. 17, n. 4, p. 482-488, Dec. 2012. Disponível em: <http://www.scielo.br/scielo.php?script=sci_arttext\&pid=S1516$80342012000400020 \&$ Ing=en\&nrm=iso>. Acesso em 10 jan. 2021. 